\section{PSICOLOGÍA IBEROAMERICANA}

\section{Psicología lberoamericana}

ISSN: 1405-0943

psicología.iberoamericana@uia.mx

Universidad Iberoamericana, Ciudad de

México

México

Luna Bernal, Alejandro César A.; Laca Arocena, Francisco A.; Mejía Ceballos, Juan Carlos Bienestar Subjetivo y Satisfacción con la Vida de Familia en Adolescentes Mexicanos de Bachillerato

Psicología Iberoamericana, vol. 19, núm. 2, julio-diciembre, 2011, pp. 17-26

Universidad Iberoamericana, Ciudad de México

Distrito Federal, México

Disponible en: http://www.redalyc.org/articulo.oa?id=133921440003

Cómo citar el artículo

Número completo

- Más información del artículo

Página de la revista en redalyc.org

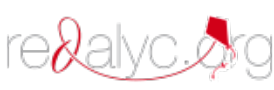

Sistema de Información Científica

Red de Revistas Científicas de América Latina, el Caribe, España y Portugal

Proyecto académico sin fines de lucro, desarrollado bajo la iniciativa de acceso abierto 


\title{
Bienestar Subjetivo y Satisfacción con la Vida de Familia en Adolescentes Mexicanos de Bachillerato
}

\author{
Subjective Well-being and Satisfaction with Family Life in Mexican Adolescents \\ High-school Students
}

\author{
Alejandro César A. Luna Bernal* \\ Universidad de Guadalajara, México \\ Francisco A. Laca Arocena* * \\ Universidad de Colima, Colima, México \\ Juan Carlos Mejía Ceballos*** \\ Universidad del País VAsco
}

\section{RESUMEN}

El objetivo del presente estudio fue examinar la relación entre el Bienestar Subjetivo y la Satisfacción Familiar en adolescentes, desde la perspectiva de la Psicología Positiva. La muestra $(N=580)$ se compuso de estudiantes de bachillerato con edades entre 15 y 19 años, quienes respondieron la Escala Multidimensional para la Medición del Bienestar Subjetivo de Anguas-Plata y Reyes-Lagunes (Anguas, 2000, 2001), la Escala de Satisfacción con Aspectos de la Vida (basada en Diener, Emmons, Larsen \& Griffin, 1985; Diener, Lucas, Oishi \& Suh, 2002; Oishi, Diener, Suh \& Lucas, 1999) y la Escala de Satisfacción con la Vida de Familia (basada en Diener et al., 1985; Laca, Verdugo \& Guzmán, 2005). Entre los resultados se encontró que la Satisfacción familiar correlacionó positivamente con diversos componentes de la Satisfacción con la vida y del Afecto positivo, mientras que lo hizo de manera negativa con los componentes del Afecto negativo. Se discuten estos y otros resultados, mostrando la pertinencia de un abordaje de la adolescencia desde la Psicología Positiva.

Descriptores: Adolescencia, psicología positiva, satisfacción familiar, emociones positivas.

\section{ABSTRACT}

The objective of this study was to examine the relationship between Subjective Well-being and Family satisfaction in adolescents, from the perspective of Positive Psychology. The sample $(N=580)$ was composed by high-school students 15 to 19 years old. They responded the Anguas-Plata and Reyes Lagunes' Multidimensional Scaling for the Measurement Subjective Well-Being (Anguas, 2000, 2001), the Domain Life Satisfaccion Scale (based in Diener, Emmons, Larsen \& Griffin, 1985; Diener, Lucas, Oishi \& Suh, 2002; Oishi, Diener, Suh \& Lucas, 1999) and the Satisfaction with Family Life Scale (based in Diener et al., 1985; Laca, Verdugo \& Guzmán, 2005). Among the results, the family satisfaction correlated positively with various components of the Satisfaction with life and Positive affect, whereas it correlated negatively with the components of Negative affect. Discusses these and other results showing the relevance of an approach of adolescence from the Positive Psychology.

Key words: Adolescence, Positive Psychology, Family Satisfaction, positive emotions

\footnotetext{
* Para correspondencia: Alejandro César Antonio Luna Bernal. Departamento de Filosofía, Universidad de Guadalajara. Av. de los Maestros y Mariano Bárcenas, Colonia Alcalde Barranquitas, 44260, Guadalajara, Jalisco. Teléfono: 0133 38193377. Correo electrónico: aluna642@hotmail.com

** Para correspondencia: Francisco Augusto Vicente Laca Arocena. Facultad de Psicología, Universidad de Colima. Av. Universidad 333, Colonia Las Víboras, 28040. Colima, Colima. Teléfono: 01312 3161091. Correo electrónico: francisco_laca@ucol.mx

*** Para correspondencia: Juan Carlos Mejía Ceballos. Departamento de Procesos Psicológicos Básicos, Facultad de Psicología, Universidad del País Vasco. Av. Tolosa 70, 20018, San Sebastián, Guipúzcoa (España).Correos electrónicos: oneyonka@hotmail.com y pbpgorej@ss.ehu.es
} 
BIENESTAR SUBJETIVO Y SATISFACCIÓN CON LA VIDA DE FAMILIA EN ADOLESCENTES MEXICANOS DE BACHILLERATO

La concepción de la adolescencia como una etapa de "tormenta y tensión" (Hall, 1904) continúa con una fuerte presencia en la cultura popular y en los medios masivos de comunicación, a pesar de la continua evidencia que la investigación psicológica ha venido acumulando, al menos desde los años sesenta del siglo XX, en apoyo de una interpretación más optimista (Alonso, 2005; Bandura, 1964; Hoffman, Paris \& Hall, 1995; Oliva, 2003, 2006; Smetana, Campione-Barr \& Metzger, 2006; Steinberg, 2001).

Más allá de la controversia sobre tormenta y tensión, la investigación actual parece orientarse a identificar factores que pueden afectar la salud y el bienestar del adolescente y su familia, al reconocer que el grado de turbulencia con que se vive dicha etapa dependerá de diferencias en los grados de vulnerabilidad y habilidad para afrontarlos (Berzonsky, 1982; Fuentes, Motrico \& Bersabé, 2003). Se distingue, asimismo, entre las manifestaciones atribuibles al periodo y las que podrían entenderse como psicopatológicas (Álvarez \& Vargas, 2002; González-Forteza, Jiménez-Tapia, Ramos-Lara \& Wagner, 2008; Siverio \& García, 2007).

En esta sintonía, se ha considerado conveniente enfocar el estudio de la adolescencia desde una perspectiva que equilibre el análisis de los malestares y las potencialidades (Contini, 2006), manteniendo en todo tiempo la conciencia de cómo las propias maneras de mirar a los adolescentes podrían condicionar a los abordajes teóricos como a las estrategias de intervención (Funes, 2003). A pesar de ello, la investigación sobre aspectos positivos o saludables del desarrollo adolescente continúa como una área emergente, enfocada a estudiar problemas del comportamiento (Oliva, 2003; Steinberg, 2001). Por ello, el presente estudio propone una aproximación a la adolescencia desde la perspectiva de la Psicología Positiva (Cuadra \& Florenzano, 2003; Kesebir \& Diener, 2008), examinando la relación entre el Bienestar Subjetivo y los juicios que los adolescentes de bachillerato hacen sobre su grado de satisfacción con su vida de familia.

En un extenso estudio sobre el significado del Bienestar Subjetivo en la cultura mexicana, Anguas (2000, 2001) identificó dos componentes del cons- tructo, en congruencia con la mayor parte de la investigación en este campo: por un lado, el emocional, compuesto por afectos positivos y negativos experimentados por el sujeto y, por otro, el cognitivo, al cual se ha denominado Satisfacción con la Vida, ya que hace referencia a un proceso de juicio mediante el cual los individuos valoran globalmente sus vidas sobre la base de su propio conjunto único de criterios (Pavot, Diener, Randall \& Sandvik, 1991). Tomando en cuenta ambos componentes, se ha afirmado que un sujeto posee un alto Bienestar Subjetivo si expresa satisfacción con su vida, frecuentes emociones positivas y poco frecuentes emociones negativas (Atienza, Pons, Balaguer \& García-Merita, 2000; Díaz, 2001).

Con relación a la familia, diversos estudios confirman que actualmente posee, un significado relevante para la mayor parte de la población (Del Valle, 2004), y que tanto los padres como los adolescentes consideran que mantener buenas relaciones familiares es un valor muy importante, situado por encima del resto de valores deseables (Megías, 2003). Según Fuentes et al. (2003), aunque los hijos inicien durante la adolescencia nuevas e importantes relaciones con amigos y parejas, mantienen los lazos afectivos con sus padres y éstos continúan siendo una de sus principales fuentes de apego y ayuda emocional. Por ello, en la presente investigación consideramos pertinente examinar la relación entre el Bienestar Subjetivo del adolescente y su grado de Satisfacción Familiar.

Algunos esfuerzos por medir el grado de satisfacción de los sujetos hacia su familia han abarcado el desarrollo de instrumentos desde la perspectiva de enfoques teóricos particulares (Musitu, Buelga, Lila \& Cava, 2004; OIson, 2000), o desde la identificación de estados emocionales (Barraca, 1999; Luengo \& Román, 2006; Velasquez \& Pedrao, 2005); sin embargo, ninguno de estos instrumentos permite estudiar el juicio global que el adolescente hace de qué tan satisfecho está de la vida con su familia sobre la base de su subjetivo conjunto único de criterios. En esta investigación consideramos, en cambio, que el preguntar al sujeto por una valoración global de su vida con su familia, desde esta perspectiva subjetiva y cognitiva, nos aportará una información que no se puede obtener a través de las medidas de los estados emocionales o de ciertos criterios de satisfacción definidos de antemano por el investigador. 
Debido a la ausencia en la literatura de alguna escala multi-ítem diseñada para medir la Satisfacción con la Vida de Familia desde esta perspectiva global, cognitiva y subjetiva, es que los autores decidimos llevar a cabo una adaptación de la Escala de Satisfacción con la Vida (Satisfaction with Life Scale, SWLS) de Diener, Emmons, Larsen y Griffin (1985), uno de los instrumentos mayormente conocidos y utilizados en el campo de la Psicología Positiva, que mide el componente cognitivo del Bienestar Subjetivo, refiriendo cada uno de los ítems de dicha escala no a la vida en general de los participantes sino a la vida con su familia. Para ello, nos apoyamos en la traducción al castellano llevada a cabo por Laca, Verdugo y Guzmán (2005).

De esta manera, se planteó como objetivo examinar las relaciones entre la Satisfacción con la Vida de Familia del adolescente y su Bienestar Subjetivo, considerando los componentes afectivos y cognitivos de este último. Nuestra hipótesis es que debe haber correlaciones positivas estadísticamente significativas entre la Satisfacción con la Vida de Familia del adolescente y su Satisfacción con la Vida en general, así como entre aquélla y las experiencias de Afecto Positivo. Al contrario, esperamos hallar correlaciones negativas entre la Satisfacción con la Vida de Familia y los componentes del Afecto Negativo. Adicionalmente, nos proponemos analizar la diversa fuerza en que la Satisfacción con la vida de familia correlacionaría con los diversos componentes del Bienestar Subjetivo. Además, y con el fin de ponderar la posible incidencia del sexo en las variables de estudio, se planteó identificar posibles diferencias por sexo en éstas.

Consideramos que el estudio de las señaladas correlaciones y diferencias por sexo podría aportar datos relevantes para la discusión sobre la manera en que uno de los aspectos positivos de la experiencia de vivir en familia (la Satisfacción Familiar) podría estar vinculado a uno de los aspectos positivos de la existencia de los adolescentes (su Bienestar Subjetivo). Lo anterior podría aportar datos a favor de la tesis de que la familia continúa siendo, durante la adolescencia, uno de los principales aspectos del Bienestar Subjetivo, no obstante que los individuos inicien nuevas e importantes relaciones con amigos y parejas durante este periodo. Finalmente, consideramos que la relevancia de los resultados del presente estudio podría contribuir al de- sarrollo de una línea de investigación sobre la adolescencia desde la perspectiva de la Psicología Positiva.

\section{MÉTODO}

\section{Participantes}

La muestra $(N=580)$ estuvo compuesta por estudiantes mexicanos de bachillerato con edades entre 15 y 19 años $(M=16.45, D T=1.02)$, de los cuales $280(48.3 \%)$ fueron hombres y 296 (51.0\%) mujeres. Los participantes pertenecían a cuatro escuelas privadas $(n=329$; $56.7 \%$ ) y dos públicas $(n=251 ; 43.3 \%)$ de la zona metropolitana de Guadalajara, Jalisco y se encontraban distribuidos en los seis niveles de bachillerato.

\section{Instrumentos}

Escala Multidimensional para la Medición del Bienestar SubjetivodeAnguas-PlatayReyes-Lagunes(EMMBSAR). Se compone de dos escalas: a) Experiencia Emocional (30 ítems) con dos subescalas, Afecto Positivo y Afecto Negativo; y, b) Evaluación Afectivo-Cognitiva de la Vida o Satisfacción con la Vida (50 ítems) (Anguas, 2000, 2001). En la primera, el participante debe indicar con qué frecuencia e intensidad ha experimentado en el último mes determinadas emociones positivas ("afecto", "alegría", "dicha", entre otras); o negativas (como "tristeza”, “angustia” o "depresión”) en una escala Likert de siete puntos. En la segunda, debe valorar qué tan satisfecho está con aquél aspecto de la vida que enuncia el reactivo en una escala tipo Likert pictórico de siete puntos. Los índices de confiabilidad alfa reportados por Anguas (2000) fueron de $.85, .85$ y .94, respectivamente.

Escala de Satisfacción de Aspectos de la Vida (ESAV). Basada en Diener et al. (1985), Oishi, Diener, Suh y Lucas (1999), y Diener, Lucas, Oishi y Suh (2002), tiene por objeto medir qué tan satisfecho se juzga un sujeto con relación a aspectos de la vida (Domain Satisfaction), tales como la familia, el dinero e ingresos, los amigos, el trabajo y la salud. En una escala de 1 a 10, donde 1 significa "muy insatisfactoria" y 10 significa "muy satisfactoria", el participante evalúa su grado de satisfacción en cada uno de los mencionados aspectos. Como se señalará en el apartado de resultados, la validación factorial realizada en el presente estudio dio por resultado una adecuada solución monofactorial con un índice de confiabilidad alfa de .79. 
Escala de Satisfacción con la Vida de Familia (ESVF). Basada en Diener et al. (1985) y Laca et al. (2005), informa por autorreporte el grado de satisfacción que un individuo juzga tener en la vida con su familia, sobre la base de su propio conjunto único de criterios. En una escala tipo Likert, que va desde " 1 = en total desacuerdo" hasta " 7 = totalmente de acuerdo", el participante señala su grado de conformidad con cada una de las cinco reactivos del cuestionario. Como se señalará en el apartado de resultados, la validación factorial realizada en el presente estudio dio por resultado una adecuada solución monofactorial con un índice de confiabilidad alfa de .88 .

\section{Procedimiento}

Se solicitó a los participantes su colaboración durante el horario de clases. Después de explicarles el objetivo del estudio, se les invitó a contestar con sinceridad, garantizándoles la absoluta confidencialidad de la información y su uso para fines exclusivamente científicos. Una submuestra de 412 participantes respondió conjuntamente la ESVF y la EMMBSAR, mientras que el resto $(n=169)$ contestó conjuntamente la ESVF y la ESAV.

Con el fin de estudiar la validez factorial de la ESVF y de la ESAV, se llevaron a cabo sendos procedimientos de Análisis Factorial Exploratorio examinando previamente su pertinencia a través de la medida de adecuación muestral de Kaiser-Mayer-Olkin (KMO) y de la prueba de esfericidad de Bartlett, empleando como mé- todo de extracción el de Componentes Principales. Se calcularon, de igual modo, los índices de confiabilidad alfa de Cronbach para cada factor resultante. El análisis factorial exploratorio de la ESVF fue llevado a cabo empleando los datos de la muestra total $(N=580)$.

Concluido el Análisis de Factorial Exploratorio, se llevó a cabo un estudio de diferencias por sexo, empleando la prueba $t$ de Student con el fin de identificar la posible incidencia de dicho factor en las variables de estudio. A continuación, con la finalidad de contrastar la hipótesis central del presente estudio, así como para examinar la posible vinculación entre la satisfacción familiar y el Bienestar Subjetivo del adolescente, se llevó a cabo un análisis correlacional Pearson entre los puntajes de la ESVF y los de la ESAV y EMMBSAR.

\section{RESULTADOS}

En cuanto el Análisis Factorial Exploratorio de la Escala de Satisfacción con la Vida de Familia, se obtuvieron valores adecuados en la medida Kaiser-MayerOlkin de adecuación muestral y en la prueba de esfericidad de Bartlett $(K M O=0.86$; c $2(10)=1475.97, p<$ $.01)$, indicando la pertinencia del análisis factorial. Los coeficientes de adecuación muestral oscilaron entre .83 y .90 , por lo que se consideraron adecuados. El método de extracción arrojó una adecuada solución monofactorial que explica en total $67.51 \%$ de la varianza. En la Tabla 1 se muestran los pesos factoriales, el autovalor, la varianza explicada y el índice de confiabilidad obtenidos.

Tabla 1. Factorial exploratorio de la Escala de Satisfacción con la Vida de Familia

\begin{tabular}{|c|l|c|}
\hline No. de item & \multicolumn{1}{|c|}{ Reactivo } & Factor I \\
\hline 3 & Estoy satisfecho(a) con mi vida de familia & .887 \\
\hline 2 & Las condiciones de mi vida con mi familia son excelentes & .837 \\
\hline $\mathbf{4}$ & En gran parte he logrado las cosas importantes que quería en la vida con mi familia & .816 \\
\hline $\mathbf{1}$ & En la mayor parte de los aspectos mi vida con mi familia está próxima a mi ideal & .814 \\
\hline $\mathbf{5}$ & Si pudiera vivir mi vida otra vez, no cambiaría casi nada de lo que he vivido con mi familia & .748 \\
\hline & Autovalor & 3.38 \\
\hline & Varianza explicada & $67.51 \%$ \\
\hline & Confiabilidad por alfa de Cronbach & .88 \\
\hline
\end{tabular}


Por otra parte, en el Análisis Factorial Exploratorio de la Escala de Satisfacción de Aspectos de la Vida se encontraron valores adecuados en la medida KaiserMayer-Olkin de adecuación muestral y en la prueba de esfericidad de Bartlett $\left(K M O=.80 ; \chi_{(15)}^{2}=230.12, p<\right.$ .01); así como coeficientes de adecuación muestral que oscilaron entre .76 y .85 , por lo que se consideraron adecuados. Como se muestra en la Tabla 2, el análisis arrojó una adecuada solución monofactorial que explica $48.90 \%$ de la varianza.

Tabla 2. Factorial exploratorio de la Escala de Satisfacción con Aspectos de la Vida

\begin{tabular}{|c|c|c|}
\hline $\begin{array}{c}\text { No. de } \\
\text { item }\end{array}$ & Factor I \\
\hline $\mathbf{2}$ & Dinero e ingresos & .741 \\
\hline $\mathbf{1}$ & Familia & .737 \\
\hline $\mathbf{4}$ & Trabajo & .716 \\
\hline $\mathbf{6}$ & Escuela & .702 \\
\hline $\mathbf{5}$ & Salud & .695 \\
\hline $\mathbf{3}$ & Amigos & .593 \\
\hline & Autovalor & 2.93 \\
\hline & $\begin{array}{r}\text { Varianza explicada } \\
\text { Cronbach }\end{array}$ & $48.90 \%$ \\
\hline
\end{tabular}

En la Tabla 3 pueden observarse las diferencias significativas, por sexo, para los puntajes de las escalas utilizadas en el presente estudio. Destaca la Satisfacción con la Vida de Familia, la Satisfacción con la Familia, y Atributos y logros personales, donde los hombres puntúan más alto que las mujeres; mientras que las mujeres puntúan más alto que los hombres en Interacción social expresada, Religión, Afecto Negativo y en algunos componentes tanto del Afecto Positivo como del Afecto Negativo.

Como puede observase en la misma Tabla 3, las correlaciones entre la Satisfacción con la Vida de Familia y la Satisfacción con Aspectos de la Vida resul- tan ser positivas en todos los casos, siendo más fuerte en el aspecto Familia y, en segundo lugar, en el aspecto Dinero.

Asimismo, en la Tabla 3 se observan correlaciones positivas entre Satisfacción con la Vida de Familia y los distintos componentes de la Satisfacción con Aspectos de la Vida de la EMMBSAR, siendo más fuertes las correlaciones con Clima familiar y Calidad de Vida.

Por último, en las correlaciones entre Satisfacción con la Vida de Familia y los diversos componentes de la Escala de Experiencia Emocional de la EMMBSAR, resultaron ser positivas en Afecto Positivo y negativas en Afecto Negativo, como se observa en la Tabla 3. 
Tabla 3. Diferencias por sexo y correlaciones Pearson entre Bienestar Subjetivo y Satisfacción con la Vida de Familia

\begin{tabular}{|c|c|c|c|c|c|}
\hline & Total & Hombre & Mujer & $t$ & $r^{a}$ \\
\hline Satisfacción con la Vida de Familia & $5.23(1.36)$ & $5.42(1.32)$ & $5.07(1.38)$ & $3.09 * *$ & 1.00 \\
\hline \multicolumn{6}{|l|}{ Satisfacción con Aspectos de la Vida } \\
\hline Familia & $8.36(1.74)$ & $8.72(1.64)$ & $7.79(1.77)$ & $3.38 * *$ & $.77^{* *}$ \\
\hline Dinero & $7.63(1.99)$ & $7.74(2.00)$ & $7.45(2.03)$ & ns & $.43 * *$ \\
\hline Amigos & $8.53(1.65)$ & $8.49(1.69)$ & $8.60(1.56)$ & ns & $.27 * *$ \\
\hline Trabajo & $7.18(2.44)$ & $7.38(2.30)$ & $6.78(2.72)$ & ns & $.36 * *$ \\
\hline Salud & $8.59(1.84)$ & $8.73(1.73)$ & $8.35(2.01)$ & ns & $.38 * *$ \\
\hline Escuela & $7.68(1.91)$ & $7.75(1.93)$ & $7.54(1.91)$ & ns & $.39 * *$ \\
\hline Total & $8.01(1.33)$ & $8.14(1.25)$ & $7.78(1.47)$ & ns & $.62 * *$ \\
\hline \multicolumn{6}{|c|}{ Satisfacción con la Vida (Evaluación afectivo-cognitiva de la vida) } \\
\hline Clima familiar & $5.76(1.13)$ & $5.83(1.05)$ & $5.72(1.19)$ & ns & $.69 * *$ \\
\hline Interacción social expresada & $6.16(0.76)$ & $6.03(0.72)$ & $6.25(0.78)$ & $2.91 * *$ & $.26^{* *}$ \\
\hline Atributos y logros personales & $5.91(0.92)$ & $6.03(0.81)$ & $5.83(0.98)$ & $2.12^{*}$ & $.49 * *$ \\
\hline Interacción social recibida & $6.20(0.85)$ & $6.11(0.90)$ & $6.27(0.80)$ & ns & $.27 * *$ \\
\hline Logros nacionales & $3.68(1.50)$ & $3.57(1.63)$ & $3.76(1.39)$ & ns & $.27 * *$ \\
\hline Religión & $5.07(1.53)$ & $4.81(1.63)$ & $5.26(1.42)$ & $2.98 * *$ & $.29 * *$ \\
\hline Calidad de vida & $6.10(0.93)$ & $6.16(0.78)$ & $6.06(1.02)$ & ns & $.52 * *$ \\
\hline Educación & $6.33(0.80)$ & $6.26(0.80)$ & $6.38(0.79)$ & ns & $.39 * *$ \\
\hline Economía & $5.18(1.31)$ & $5.32(1.32)$ & $5.09(1.30)$ & ns & $.34 * *$ \\
\hline Total & $5.95(0.73)$ & $5.96(0.67)$ & $5.94(0.76)$ & ns & $.57 * *$ \\
\hline \multicolumn{6}{|l|}{ Afecto Positivo } \\
\hline Afectividad positiva & $5.83(1.01)$ & $5.70(1.01)$ & $5.93(1.01)$ & $2.12^{*}$ & $.35 * *$ \\
\hline Afectividad interna positiva & $4.94(1.23)$ & $4.78(1.24)$ & $5.06(1.20)$ & $2.18^{*}$ & $.29 * *$ \\
\hline Éxito & $5.14(1.21)$ & $5.23(1.12)$ & $5.07(1.26)$ & ns & $.30 * *$ \\
\hline Hedonismo emocional & $4.78(1.40)$ & $4.91(1.35)$ & $4.68(1.42)$ & ns & $.20 * *$ \\
\hline Esperanza & $5.26(1.23)$ & $5.11(1.24)$ & $5.36(1.22)$ & $1.98^{*}$ & $.24 * *$ \\
\hline Total & $5.19(0.97)$ & $5.14(0.95)$ & $5.22(.98)$ & ns & $.34 * *$ \\
\hline \multicolumn{6}{|l|}{ Afecto Negativo } \\
\hline Afectividad negativa & $4.02(1.45)$ & $3.70(1.40)$ & $4.25(1.44)$ & $3.78 * *$ & $-.19 * *$ \\
\hline Expresividad negativa & $4.24(1.41)$ & $4.22(1.39)$ & $4.25(1.42)$ & ns & $-.33 * *$ \\
\hline Expresividad emocional interna negativa & $3.68(1.45)$ & $3.44(1.39)$ & $3.85(1.47)$ & $2.77^{* *}$ & $-.32 * *$ \\
\hline Emocionalidad negativa & $3.32(1.52)$ & $2.89(1.44)$ & $3.63(1.52)$ & $4.82^{* *}$ & $-.34^{* *}$ \\
\hline Nerviosismo & $3.96(1.38)$ & $3.85(1.32)$ & $4.03(1.42)$ & ns & $-.27^{* *}$ \\
\hline Total & $3.84(1.13)$ & $3.62(1.05)$ & $4.00(1.16)$ & $3.30 * *$ & $-.37 * *$ \\
\hline
\end{tabular}

Nota $1:{ }^{*} p<.05,{ }^{* *} p<.01$.

Nota 2: $r$ a Correlaciones con Satisfacción con la Vida de Familia 


\section{DISCUSIÓN}

Los resultados obtenidos en los Análisis Factoriales Exploratorios de la Escala de Satisfacción con la Vida de Familia (ESVF) y de la Escala de Satisfacción con Aspectos de la Vida (ESAV) fueron, en ambos casos, soluciones monofactoriales adecuadas con cargas factoriales altas para todos los ítems y buenos índices de confiabilidad. Debido a ello, pueden ser considerados como dos instrumentos confiables para ser utilizados en este campo.

En general, las puntuaciones en Satisfacción con la Vida de Familia, Satisfacción con Aspectos de la Vida, Satisfacción con la Vida y Afecto Positivo tienden a ser altos, mientras que los puntajes de Afecto Negativo suelen ser moderadamente bajos, como se observa en la Tabla 3. La única excepción a este respecto lo constituye el factor de Logros Nacionales, cuyas puntuaciones tienden a ser discretamente bajas. Tales resultados apoyan una perspectiva más equilibrada sobre la adolescencia, al mostrar en los adolescentes del presente estudio un predominio moderado de los aspectos positivos sobre los negativos del Bienestar Subjetivo. Tales hallazgos concuerdan con los resultados de Anguas (2000), que mostraron en general niveles altos de Bienestar Subjetivo, que tienden a mantenerse o incrementarse a lo largo del ciclo vital, a excepción del factor Logros Nacionales, en el que se obtuvieron sistemáticamente las puntuaciones más bajas. Este último hecho podría entenderse como la expresión de un cierto grado de descontento de la población en general, y de los adolescentes en particular, respecto de los logros gubernamentales y de la política tal como se practica actualmente en México, aunque serán necesarios estudios posteriores sobre esta cuestión con el fin de confirmar dicha interpretación.

En cuanto a las diferencias por sexo, los varones manifestaron estar más satisfechos que las mujeres tanto con sus Atributos y logros personales como con su Vida de Familia. Ello podría estar relacionado con dos elementos: por un lado, el logro de la autonomía respecto de la autoridad parental constituye una de las tareas características de la adolescencia como periodo vital (Coleman, 1994; Alonso, 2005); por otro, el relativo predominio de los roles de género tradicionales en las familias mexicanas, podría facilitar el logro de mayor autonomía por parte del adolescente varón en aquellos aspectos que tienen que ver con su futuro rol como agente proveedor y cabeza de familia. Así, combinándose ambos factores, contribuirían a que el adolescente varón percibiera mayor autonomía familiar en aquellos aspectos que se relacionan con su futuro rol de género. Ello podría explicar también porqué las adolescentes resultaron mostrarse significativamente más satisfechas que los varones en los aspectos de Interacción social expresada y Religión, ya que ambos se relacionan con rasgos tradicionales estereotípicamente atribuidos a las mujeres, como ser más sensible a las necesidades de los demás y ser más comprensivas e intuitivas (Cuadrado, 2007). Lo mismo cabe decir del aspecto afectivo, donde las mujeres mostraron mayores puntajes tanto de Afecto Negativo como del Positivo, lo que concuerda con la imagen estereotipada de mayor susceptibilidad emocional de la mujer (Valdez, Díaz y Pérez, 2005).

En el análisis correlacional cabe observar correlaciones positivas fuertes de la Satisfacción Familiar con la Satisfacción con la Vida y con la Satisfacción con Aspectos de la Vida. Este dato apoya la tesis de que la familia continúa siendo, durante la adolescencia, uno de los principales aspectos del Bienestar Subjetivo, no obstante que los individuos inicien nuevas e importantes relaciones con amigos y parejas durante este periodo (Fuentes et al., 2003; Musitu et al., 2004). Además, ello concuerda con los hallazgos de Anguas (2000), que destacan la peculiar importancia que reviste la familia como componente del Bienestar Subjetivo en la cultura mexicana.

Respecto a la relación más específica entre la Satisfacción con la Vida de Familia y los componentes cognitivos del Bienestar Subjetivo, cabe destacar el siguiente hecho. Descontando los elementos correspondientes a "Familia" de la ESAV y a "Clima familiar" de la EMMBSAR, las siguientes correlaciones más importantes de la Satisfacción Familiar se producen con Calidad de vida, Atributos y logros personales, Dinero, Educación, Escuela, Salud, Trabajo y Economía, lo que podría revelar la importancia que para el adolescente reviste la familia como proveedora de bienes de naturaleza fundamentalmente económica.

En la parte afectiva, sin embargo, observamos correlaciones de moderadas a fuertes entre la Satisfacción con la Vida de Familia y la Afectividad positiva y, en 
sentido negativo, de dicha Satisfacción Familiar con la Expresividad negativa, la Expresividad emocional interna negativa y la Emocionalidad negativa. Ello significaría que una mayor Satisfacción con la Vida de Familia estaría relacionada con mayor frecuencia e intensidad de experiencias afectivas de amor, cariño, afecto, alegría y felicidad; mientras que una menor Satisfacción con la Vida de Familia se relacionaría con mayores experiencias de coraje, enojo, frustración, desilusión, tristeza y depresión, según los composición que de dichos factores presentó Anguas (2000).

Como se puede observar, se logró cumplir con el objetivo de la presente investigación al examinar las relaciones entre la Satisfacción con la Vida de Familia del adolescente y su Bienestar Subjetivo, considerando los componentes afectivos y cognitivo de este último. Ello, mediante el análisis correlacional efectuado entre los puntajes de la ESVF, de la ESAV y de las diversas escalas y subescalas de la EMMBSAR. De la misma manera, la hipótesis planteada, según la cual habría de encontrarse una relación positiva estadísticamente significativa entre la Satisfacción con la Vida de Familia del adolescente y su Satisfacción con la Vida en general, así como entre aquélla y el Afecto Positivo (y al contrario, correlaciones negativas entre la Satisfacción con la Vida de Familia y los componentes del Afecto
Negativo), fue corroborada por los resultados de la presente investigación, tal como se aprecia en los párrafos precedentes.

En conclusión, los resultados del presente estudio apoyan la tesis de que la familia continúa siendo, durante la adolescencia, uno de los principales aspectos del Bienestar Subjetivo, no obstante que los individuos inicien nuevas e importantes relaciones con amigos y parejas durante este periodo.

Además, tales resultados muestran la viabilidad de una línea de investigación sobre la adolescencia desde la perspectiva de la Psicología Positiva, apoyando una concepción más equilibrada de este periodo del desarrollo humano que, contrastando con la imagen tradicional de la adolescencia como un periodo de "tormenta y tensión", es coherente, sin embargo, con los estudios empíricos que se han realizado en este campo en las últimas décadas.

Dentro de las limitaciones del presente estudio, cabe señalar que el mismo ha sido realizado con adolescentes estudiantes de bachillerato entre 15 y 19 años de edad, por lo cual cabría en próximos estudios extender la muestra a edades menores con el fin de estudiar estos aspectos en la adolescencia temprana, así como incluir a participantes no escolarizados.

\section{REFERENCIAS}

Alonso, P. (2005). Discrepancia entre padres e hijos en la percepción del funcionamiento familiar $y$ desarrollo de la autonomía del adolescente. (Tesis doctoral). Universidad de Valencia, Valencia, España.

Álvarez, R. \& Vargas, M. (2002). Violencia en la adolescencia. Salud en Tabasco, 8(2), 95-98.

Anguas, A. (2000). El bienestar subjetivo en la cultura mexicana. (Tesis doctoral). Universidad Nacional Autónoma de México, México.

Anguas, A. (2001). Identificación y validación del significado del bienestar subjetivo en México: fundamentos para el desarrollo de un instrumento de medición. Interamerican Journal of Psychology, 35(1), 163-183.
Atienza, F., Pons, D., Balaguer, I., \& García, M. (2000). Propiedades psicométricas de la Escala de Satisfacción con la Vida en adolescentes. Psicothema, 12(2), 314-319.

Bandura, A. (1964). The stormy decade: Fact or fiction? Psychology in the Schools, 1(3), 224-231.

Barraca, J. (1999). Claves psicológicas de la satisfacción familiar de los jóvenes. Diálogo Familia-Colegio, 212, 40-46.

Berzonsky, M. (1982). Inter- and intraindividual differences in adolescent storm and stress: a life-span developmental view. Journal of Early Adolescence, 2(3), 211-217. 
Coleman, J. (1994). Psicología de la adolescencia (3ª ed.). (Tr., A. Guerra). Madrid: Ediciones Morata. (Obra original publicada en 1980).

Cuadra, H. \& Florenzano, R. (2003). El bienestar subjetivo: hacia una psicología positiva. Revista de Psicología de la Universidad de Chile, 12(1), 83-96.

Cuadrado, I. (2007). Estereotipos de género. En J. F. Morales, M. C. Moya, E. Gaviria, e I. Cuadrado (Coords.), Psicología social (3ª ed., pp. 243-266). Madrid: McGraw Hill.

Contini, N. (2006). El paradigma salugénico: un nuevo modo de analizar el comportamiento adolescente. En N. Contini (Comp.), Pensar la adolescencia hoy. De la psicopatología al bienestar psicológico (pp. 2134). Buenos Aires: Paidós.

Del Valle, I. (2004). El futuro de la familia: la familia. Iglesia Viva. Revista de Pensamiento Cristiano, 217, $1-26$.

Díaz, G. (2001). El bienestar subjetivo. Actualidad y perspectivas. Revista Cubana de Medicina General Integral, 17(6), 572-579.

Diener, E., Emmons, R., Larsen, R. \& Griffin, S. (1985). The Satisfaction with Life Scale. Journal of Personality Assessment, 49(1), 71-75.

Diener, E., Lucas, R., Oishi, S. \& Suh, E. (2002). Looking up and looking down: weighting good and bad information in life satisfaction judgments. Personality and Social Psychology Bulletin, 28(4), 437-445.

Fuentes, M., Motrico, E. \& Bersabé, R. (2003). Estrategias de socialización de los padres y conflictos entre padres e hijos en la adolescencia. Anuario de Psicología, 34(3), 385-400.

Funes, J. (2003). ¿Cómo trabajar con adolescentes sin empezar por considerarlos un problema? Papeles del Psicólogo, 23(84), 1-8.

González-Forteza, C., Jiménez-Tapia, J., Ramos-Lara, L. y Wagner, F. (2008). Aplicación de la Escala de Depresión del Center Epidemiological Studies en adolescentes de la Ciudad de México. Salud Pública de México, 50(4), 292-299.

Hall, G. S. (1904). Adolescence: Its psychology and its relations to physiology, anthropology, sociology, sex, crime, religion and education (Vol. 1). New York: D Appleton \& Company. Recuperado el 10 de Octubre de 2010 de: http://psycnet.apa.org/books/10616/
Hoffman, L., Paris, S. \& Hall, E. (1995). Psicología del desarrollo, hoy. (Vol. 2, $6^{\mathrm{a}}$ ed.). (Tr., E. Serra). Madrid: McGraw Hill. (Obra original publicada en 1994).

Kesebir, P. \& Diener, E. (2008). In pursuit of happiness. Empirical answers to philosophical questions. Perspectives on Psychological Science, 3(2), 117-125.

Laca, F., Verdugo, L. y Guzmán, J. (2005). Satisfacción con la vida de algunos colectivos mexicanos: una discusión sobre la psicología del bienestar subjetivo. Enseñanza e Investigación en Psicología, 10(2), 325336.

Luengo, J. \& Roman, J. (2006). Estructura familiar y satisfacción parental: propuestas para la intervención. Acciones e Investigaciones Sociales, 1(extra), 455-490.

Megías, E. (2003). Comunicación y conflicto entre hijos $y$ padres. Madrid: Fundación de Ayuda contra la Drogadicción.

Musitu, G., Buelga, S., Lila, M. \& Cava, M. (2004). Familia $y$ adolescencia. Un modelo de análisis e intervención psicosocial. Madrid: Síntesis.

Oishi, S., Diener, E., Shu, E., \& Lucas, R. (1999). Values as moderador in subjective well-being. Journal of Personality, 67(1), 157-184.

Oliva, A. (2003). Adolescencia en España a principios del siglo XXI. Cultura y Educación, 15(4), 373-383.

Oliva, A. (2006). Relaciones familiares y desarrollo adolescente. Anuario de Psicología, 37(3), 209-223.

Olson, D. (2000). Circumplex model of marital and family systems. Journal of Family Therapy, 22, 144-167.

Pavot, W., Diener, E., Randall, C. \& Sandvik, E. (1991). Further validation on the satisfaction with life Scale: evidence to the cross-method convergence of wellbeing measures. Journal of Personality Assessment, 5(1), 149-161.

Siverio, M. \& García, M. (2007). Autopercepción de adaptación y tristeza en la adolescencia. La influencia del género. Anales de Psicología, 23(1), 41-48.

Smetana, J., Campione-Barr, N. \& Metzger, A. (2006). Adolescent development in interpersonal and societal contexts. Annual Review of Psychology, 57, 255284.

Steinberg, L. (2001). We know some things: parent-adolescent relationships in retrospect and prospect. Journal of Research on Adolescence, 11(1), 1-9.

Valdez, J., Díaz, R. \& Pérez, M. (2005). Los hombres y las mujeres en México: Dos mundos distantes y comple- 
mentarios. México: Universidad Autónoma del Estado de México.

Velasquez, D. \& Pedrao, L. (2005). Satisfacción personal del adolescente adicto a las drogas en el ambiente familiar durante la fase de tratamiento en un instituto de salud mental. Revista Latinoamericana de Enfermagem, 13(no. Especial), 836-844.

Fecha de recepción: Mayo 2011 Fecha de publicación: Diciembre 2011 